\title{
Editorial
}

\section{Advanced Issues on Topic Detection, Tracking, and Trend Analysis for Social Multimedia}

\author{
Seungmin Rho, ${ }^{1}$ Wenny Rahayu, ${ }^{2}$ and Uyen Trang Nguyen ${ }^{3}$ \\ ${ }^{1}$ Department of Multimedia, Sungkyul University, Anyang-si, Gyeonggi-do 430-742, Republic of Korea \\ ${ }^{2}$ Department of Computer Science and Computer Engineering, La Trobe University, Bundoora, VIC 3086, Australia \\ ${ }^{3}$ Department of Computer Science and Engineering, York University, Toronto, ON, Canada M3J 1P3 \\ Correspondence should be addressed to Seungmin Rho; pc.seungminrho@gmail.com
}

Received 16 March 2015; Accepted 16 March 2015

Copyright (C) 2015 Seungmin Rho et al. This is an open access article distributed under the Creative Commons Attribution License, which permits unrestricted use, distribution, and reproduction in any medium, provided the original work is properly cited.

Introduction. In recent years, the smartphone boom has made social media monitoring of news, blogs, Twitter, and Facebook a social phenomenon. Twitter is an online social networking and microblogging service that has gained worldwide popularity with over 500 million active users as of 2012 . Even though one tweet may contain at most 140 characters, the number of tweets that are generated daily is enormous and hence they, collectively, could give important clues to several issues such as public opinions, current trends, and burst keywords. If detecting the information could be successfully done, various services related to the information, such as multimedia contents recommendation and trend contents visualization, can be provided. So far, many studies have been carried out for this goal. Therefore, we are looking for efficient and effective meaningful information such as topic and trend detecting/tracking algorithms in SNS (social network services) big data.

Each submitted manuscript was blindly reviewed by at least three reviewers consisting of guest editors and external reviewers. After two review processes, nine manuscripts were selected to be included in this special issue. In Summaries of Selected Manuscripts, we briefly summarize these manuscripts.

Summaries of Selected Manuscripts. The article entitled "Study on Strengthening Plan of Safety Network CCTV Monitoring by Steganography and User Authentication" by J. O. Park and S. Kim proposes an image checking technique through steganography to ensure the security of the image transfer process and user authentication protocol to block malicious users in network CCTV environments.

In the paper entitled "Supporting Image Search with Tag Clouds: A Preliminary Approach," F. Guerra et al. introduce a novel tag-based system supporting the search of images published with some textual information. For two particular use cases, they also implement MediaPresenter which is a system for (i) the creation of multimedia presentations and (ii) an image search engine for Twitter.

In the paper entitled "Performance Comparison of OpenMP, MPI, and MapReduce in Practical Problems," S. J. Kang et al. review existing parallel computing models and present performance studies of three widely recognized parallel programming frameworks: OpenMP, MPI, and MapReduce. The comparative study was conducted for two problem sets: the all-pair shortest path problem and a joint problem for large data sets.

The paper entitled "Security Requirements for Multimedia Archives” by S. B. Park presents security issues related to digital archive systems. The author discusses security problems of cryptographic primitives and lifetime of cryptographic keys for long term preservation. For long term security, the author suggests periodic updates of cryptographic messages and keys. The study is not to solve those problems but rather to enumerate the requirements which must be considered for secure digital archiving systems.

In the paper entitled "Coevolution of Artificial Agents using Evolutionary Computation in Bargaining Game," S. Lee investigates the interaction and coevolutionary process 
among heterogeneous artificial agents using evolutionary computation (EC) in the bargaining game. The three kinds of EC based agents are compared in the bargaining game: genetic algorithm, particle swarm optimization, and differential evolution. The game performance with regard to payoff through the interaction and coevolution of agents is studied. The study demonstrates that the particle swarm optimization approach is superior to the other algorithms in the bargaining game.

In the paper entitled "Preprocessing Techniques for HighEfficiency Data Compression in Wireless Multimedia Sensor Networks," J. Park and J. Yoo propose novel preprocessing techniques for high-efficiency data compression in wireless multimedia sensor networks. The proposed techniques consider the characteristics of sensed multimedia data to perform the first stage preprocessing by deleting the low priority bits that do not affect the image quality. The second stage preprocessing is also performed for the undeleted high priority bits. These preprocessing techniques significantly reduce the size of multimedia data to be compressed. To show the superiority of the proposed techniques, an existing multimedia data compression scheme with and without our preprocessing techniques was implemented. The experimental results have shown that the proposed techniques increase the compression ratio while reducing the number of compression operations compared to the existing compression scheme without the preprocessing techniques.

The paper entitled "Discovering Congested Routes Using Vehicle Trajectories in Road Networks" by K. S. Bok et al. proposes a new discovering scheme of congested routes through the analysis of vehicle trajectories in a road network. The proposed scheme computes the complexity value of each road segment according to different directions and detects congested routes of a road network by clustering congested road segments within each time interval. To show the superiority of the proposed scheme, a performance evaluation was performed by comparing the proposed scheme with the existing scheme NETSCAN. The experimental results show that the proposed scheme can efficiently discover congested routes in different directions better than the existing scheme.

In the paper entitled "An Empirical Analysis of Technology Transfer of National R\&D Projects in South Korea," M.-S. Kim et al. examine the outcomes of national R\&D projects by the types of R\&D organizations and by the types of $\mathrm{R} \& \mathrm{D}$ and analyze the factors influencing the outcomes. Their analysis indicates that there were significant differences between government funded institutions and universities and between basic and applied R\&D. The study has found that government funded institutions did not necessarily perform better than universities in terms of the quantity of technology transfer.

In the paper entitled "Development of Ontology and 3D Software for the Diseases of Spine," S. Lee et al. introduce an e-Spine project carried out by the Korea Institute of Science and Technology Information (KISTI) that deals with spinal diseases to prepare for an ageing society. They built an ontology of the spine with links to the causes, symptoms, and methods of treatment of frequently encountered spinal diseases among Koreans and anatomical information.
The completed spinal ontology expresses anatomical connections of the parts of the spine and their vertical relationships as well as information on spinal diseases. It is easy to understand the spinal structure and diseases by conceptualizing the anatomical structure of the spine and showing it in $3 \mathrm{D}$ images.

\section{Acknowledgments}

Our special thanks go to the editorial board members of this journal for their valuable supports throughout the preparation and publication of this special issue. We would like to thank all authors for their contributions to this special issue. We also extend our thanks to the external reviewers for their time and efforts in reviewing the manuscripts.

Seungmin Rho Wenny Rahayu Uyen Trang Nguyen 

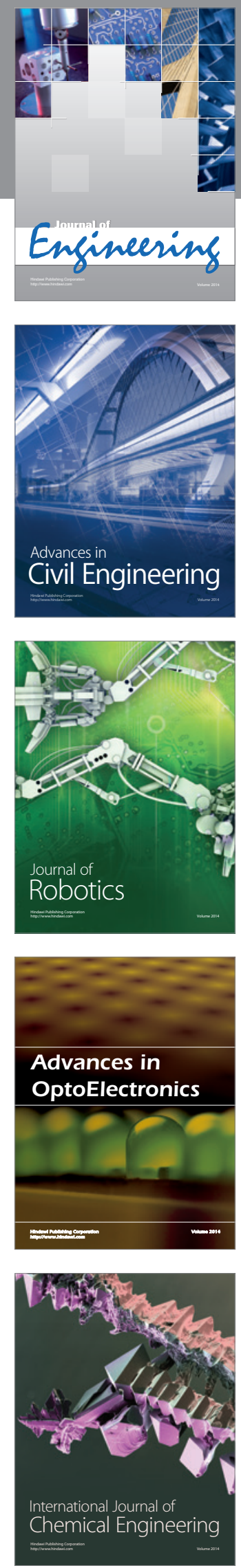

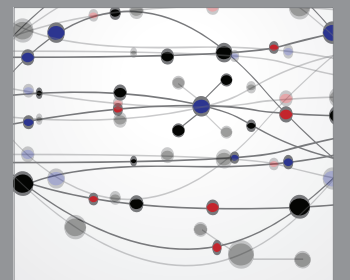

The Scientific World Journal
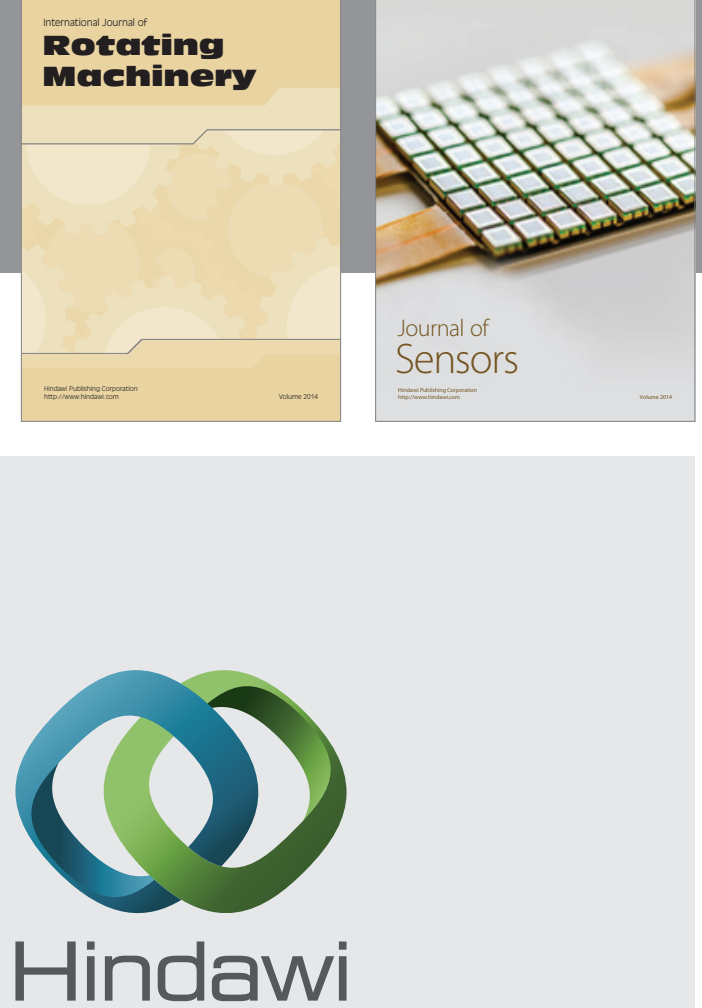

Submit your manuscripts at http://www.hindawi.com
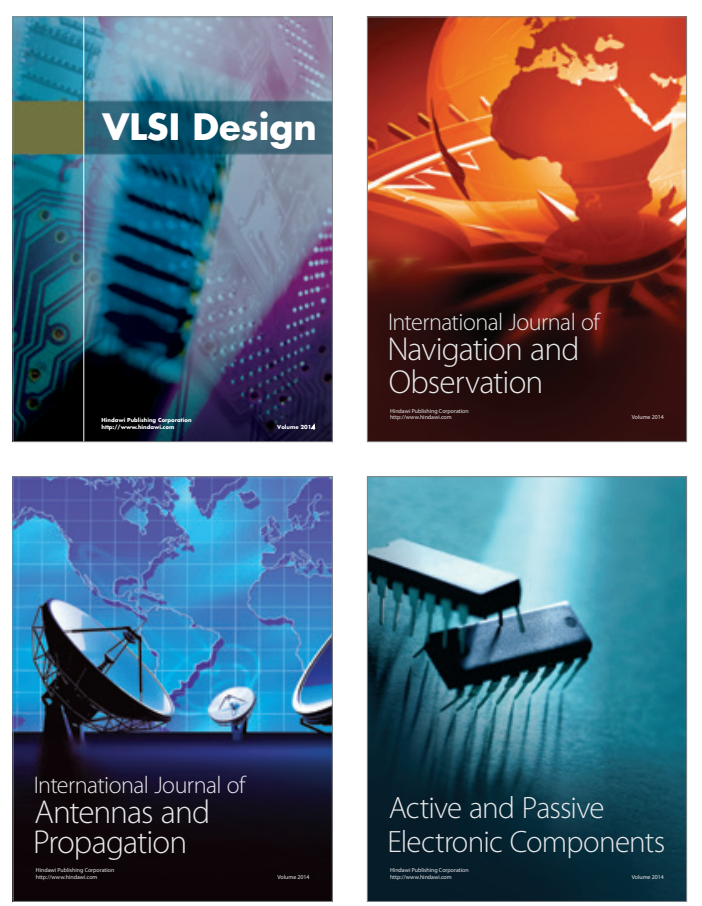
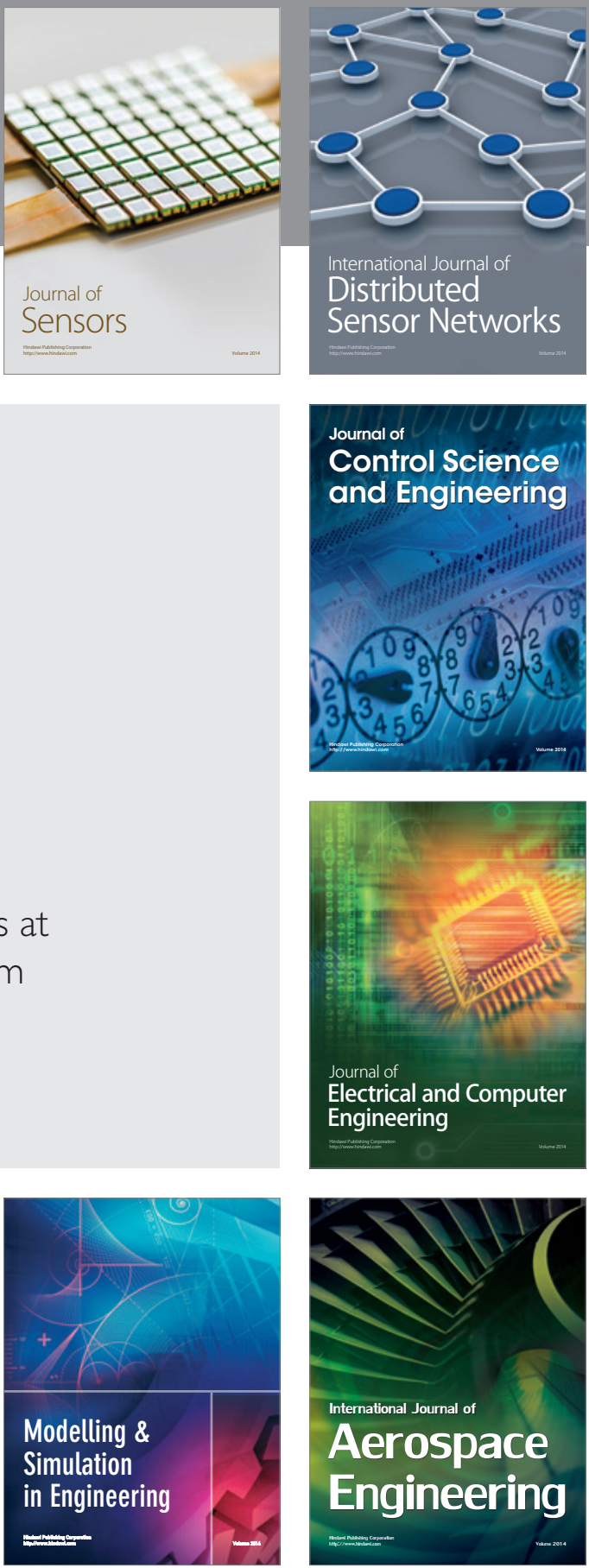

Journal of

Control Science

and Engineering
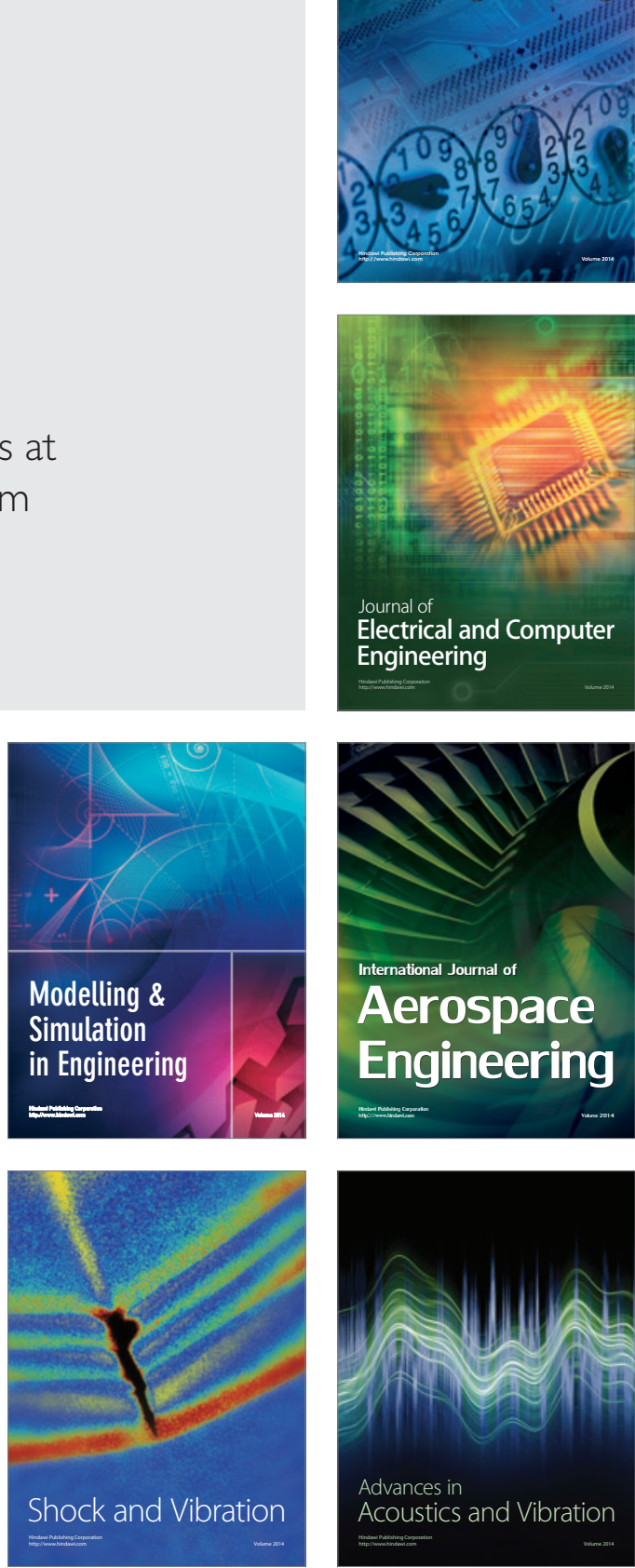